\title{
Candidate High Redshift Clusters of Dusty Galaxies from Herschel and Planck
}

\author{
D. L. Clements ${ }^{1}$, J. Greenslade ${ }^{2}$, the HerMES \& H-ATLAS Consortia \\ ${ }^{1}$ Physics Department, Imperial College, Prince Consort Road, London SW7 2AZ, UK \\ email: d.clements@imperial.ac.uk \\ ${ }^{2}$ Physics Department, Imperial College, Prince Consort Road, London SW7 2AZ, UK \\ email: j.greenslade14@imperial.ac.uk
}

\begin{abstract}
By examining Herschel images in the HerMES \& H-ATLAS surveys at the position of Planck Catalog of Compact Source sources we are able to determine the nature of the Planck sources. Most are simply nearby, known, dusty galaxies, while others are foreground galactic 'cirrus' dust. About 11\% of sources, though, appear to be groups or clumps of fainter Herschel objects. Followup of a number of these indicates that they are galaxy clusters or protoclusters at $\mathrm{z} \sim 1-3$ that contain a number of galaxies undergoing contemporaneous massive starbursts. These sources present challenges for current galaxy \& cluster formation/evolution models.
\end{abstract}

Keywords. Galaxy clusters, Starburst Galaxies, Galaxy Formation

\section{The Search for Dusty Clusters of Galaxies}

The early history of galaxy clusters is currently a poorly constrained aspect of galaxy formation. Normal X-ray, optical/near-IR and Sunyaev-Zeldovich approaches to finding galaxy clusters generally become ineffective at redshifts $>\sim 1.5$. Theoretical models (eg. Granato et al., 2015) suggest that galaxies in a forming cluster will undergo nearsimultaneous massive bursts of star formation. Such objects will have significant far-IR \& submm luminosities and thus might be detectable in large area Herschel surveys and in the Planck all sky surveys. Negrello et al. (2005), for example, suggested such objects would be detectable by Planck as compact sources which would break up into overdensities of individual objects when observed at the higher resolutions available to Herschel.

We searched for such objects by examining the H-ATLAS \& HerMES Herschel surveys at positions matching those of compact sources in the Planck ERCSC and PCCS1 catalogs. We find that these objects divide easily into four classes: known sources $(66 \%)$ such as IRAS galaxies; galactic cirrus (24\%); protocluster candidates (7.5\%); and lens candidates $(2.5 \%)$.

Followup observations of our first candidates using a combination of optical, near-IR and submm data, using both archival and dedicated observations, have confirmed the cluster nature of the first four candidates found (Clements et al., 2014). Followup observations of our larger sample continue. Further modelling of such objects using modern N-body/hydro codes combined with radiative transfer modelling to account for dust absorption and re-emission (Granato et al., 2015) suggests that the observed number counts we are finding for these objects are higher than predicted.

\section{References}

Clements, D. L., et al. 2014, MNRAS, 439, 1193

Granato, G. L., et al. 2015, MNRAS, 450, 1320

Negrello, M., et al. 2005, MNRAS, 358, 869 